\title{
Estados localizados no modelo de Kronig-Penney fracionário com defeito
}

\author{
Arianne Vellasco-Gomes ${ }^{1}$ \\ Universidade Federal de Roraima, Escola Agrotécnica. \\ Alexys Bruno-Alfonso ${ }^{2}$ \\ Universidade Estadual Paulista, Departamento de Matemática. \\ Rubens de Figueiredo Camargo ${ }^{3}$ \\ Universidade Estadual Paulista, Departamento de Matemática.
}

Resumo São calculados e analisados os níveis de energia e as funções de onda para o modelo de Kronig-Penney fracionário com defeito e derivada de Riesz. Na ausência do defeito, o potencial consiste de deltas de Dirac de igual intensidade, uniformemente espaçadas. O defeito consiste em alterar a intensidade do potencial de um dos deltas de Dirac, preservando a simetria de inversão. Os estados da partícula podem ser localizados ou não. A pesquisa é dedicada ao estudo dos estados localizados, mediante o método da transformada de Fourier. O resultado mais importante é que, no caso fracionário, as funções de onda decaem em forma de lei de potência, com expoente aproximadamente igual a $-(\alpha+1)$.

Palavras-chave. Cálculo fracionário, equação de Schrödinger fracionária, transformada de Fourier, comportamento assintótico.

\section{Introdução}

A equação de Schrödinger fracionária independente do tempo, para a função de onda $\psi(x)$, é dada por $[2,3]$

$$
-C_{\alpha} D^{\alpha} \psi(x)+V(x) \psi(x)=E \psi(x) .
$$

Aqui o operador fracionário $D^{\alpha}$ é derivada de Riesz. O parâmetro $C_{\alpha}$ caracteriza a inércia da partícula, $V(x)$ é a energia potencial e $E$ é a energia total da mesma. Para o modelo de Kronig-Penney fracionário com defeito na origem [1], utilizamos o potencial da forma

$$
V(x)=V_{0} \delta\left(\frac{x}{a}\right)+\sum_{m \in \mathbb{Z}^{*}} V \delta\left(\frac{x}{a}-m\right),
$$

em que $V$ é a intensidade do potencial de todos os poços quânticos, excetuando o poço quântico situado na origem, que tem intensidade $V_{0}$.

\footnotetext{
${ }^{1}$ arianne.vellasco@ufrr.br

2 alexys.bruno-alfonso@unesp.br

3 rubens.camargo@unesp.br
} 
Substituindo o potencial na Eq. (1) e aplicando a transformada de Fourier em ambos os lados da equação, obtemos

$$
\left(E-C_{\alpha}|k|^{\alpha}\right) \phi(k)=a \Delta V \psi(0)+a V \sum_{m \in \mathbb{Z}} e^{-i k m a} \psi(m a) .
$$

Aqui $\Delta V=V_{0}-V$ quantifica o defeito do potencial, aquilo que quebra a sua periodicidade.

Os valores da função de onda nas posições dos poços quânticos podem ser calculados pela transformada inversa de Fourier, ou seja,

$$
\psi(m a)=\frac{1}{2 \pi} \int_{-\infty}^{+\infty} e^{i k m a} \phi(k) d k
$$

Substituindo esta expressão no segundo termo do lado direito da Eq. (3), obtemos

$$
\left(E-C_{\alpha}|k|^{\alpha}\right) \phi(k)=\Delta V \psi(0)+V S(k),
$$

em que

$$
S(k)=\sum_{n \in \mathbb{Z}} \phi\left(k+\frac{2 \pi n}{a}\right) .
$$

Isolando $\phi(k)$ da equação Eq. (5), temos

$$
\phi(k)=\frac{a \Delta V \psi(0)}{E-C_{\alpha}|k|^{\alpha}}+\frac{V S(k)}{E-C_{\alpha}|k|^{\alpha}}+P(k),
$$

na qual $P(k)$ assumirá valores distintos, dependendo do valor da energia, a saber,

$$
P(k)= \begin{cases}A_{+} \delta\left(k-\left(\frac{E}{C_{\alpha}}\right)^{1 / \alpha}\right)+A_{-} \delta\left(k+\left(\frac{E}{C_{\alpha}}\right)^{1 / \alpha}\right), & \text { se } E>0 \\ 0, & \text { se } E<0,\end{cases}
$$

em que $A_{+}$e $A_{-}$são constantes [2]. A contribuição de $P(k)$ para $\psi(x)$ é

$$
\frac{1}{2 \pi} \int_{-\infty}^{+\infty} P(k) e^{i k x} d k= \begin{cases}\frac{A_{+}}{2 \pi} e^{i x\left(E / C_{\alpha}\right)^{1 / \alpha}}+\frac{A_{-}}{2 \pi} e^{-i x\left(E / C_{\alpha}\right)^{1 / \alpha}}, & \text { se } E>0, \\ 0, & \text { se } E<0 .\end{cases}
$$

Para $E>0$, temos uma combinação linear de ondas harmônicas que se estendem ao infinito. Como estamos à procura de estados localizados, escolhemos $A_{-}=A_{+}=0$, ou seja, $P(k)=0$. Então,

$$
\phi(k)=\frac{a \Delta V \psi(0)}{E-C_{\alpha}|k|^{\alpha}}+\frac{V S(k)}{E-C_{\alpha}|k|^{\alpha}} .
$$

em que

$$
S(k)=\sum_{n \in \mathbb{Z}} \phi\left(k+\frac{2 \pi n}{a}\right)=\sum_{n \in \mathbb{Z}}\left[\frac{a \Delta V \psi(0)}{E-C_{\alpha}\left|k+\frac{2 \pi n}{a}\right|^{\alpha}}+\frac{V S\left(k+\frac{2 \pi n}{a}\right)}{E-C_{\alpha}\left|k+\frac{2 \pi n}{a}\right|^{\alpha}}\right] .
$$


Calculando a transformada de Fourier inversa de $\phi(k)$ dada pela Eq. (10), obtemos que a função de onda dos estados localizados é

$$
\psi(x)=\frac{a \Delta V \psi(0)}{2 \pi} \int_{-\infty}^{+\infty} \frac{e^{i k x} d k}{\left(E-C_{\alpha}|k|^{\alpha}\right)(1-V g(E, k))} .
$$

Convenientemente, trabalhamos com a forma adimensional da função de onda, dada por

$$
\chi(\xi)=\psi(x) \sqrt{a}=\Delta v \chi(0) \int_{-\infty}^{+\infty} \frac{e^{2 \pi i \kappa \xi} d \kappa}{\left(\epsilon-|\kappa|^{\alpha}\right)(1-v G(\epsilon, \kappa))},
$$

em que $\xi=x / a, v=V / K_{\alpha}, \Delta v=\Delta V / K_{\alpha}, \chi(0)=\psi(0) \sqrt{a}, \epsilon=E / K_{\alpha}$ e $\kappa=k /(2 \pi / a)$, $K_{\alpha}=C_{\alpha}\left(\frac{2 \pi}{a}\right)^{\alpha}$ e $G(\epsilon, \kappa)=\sum_{n \in \mathbb{Z}} \frac{1}{\epsilon-|\kappa+n|^{\alpha}}$.

Ainda é necessário especificar o valor de $\chi(0)$. Seu valor absoluto é calculado a partir da condição de normalização, que é dada por $\int_{-\infty}^{+\infty}|\psi(x)|^{2} d x=\int_{-\infty}^{+\infty}|\chi(\xi)|^{2} d \xi=1$. Então, obtemos

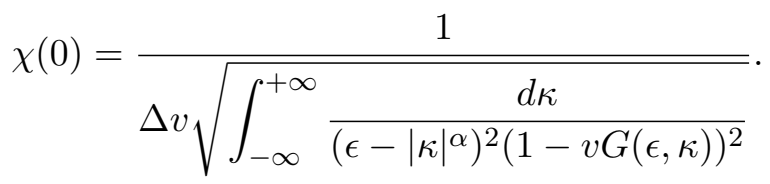

Considerando a Eq. (13) para $\xi=0$, temos que energia de todo estado localizado deve satisfazer

$$
\frac{1}{\Delta v}=\int_{-\infty}^{+\infty} \frac{d \kappa}{\left(\epsilon-|\kappa|^{\alpha}\right)(1-v G(\epsilon, \kappa))} .
$$

Consequentemente, a função de onda se reduz à forma

$$
\chi(\xi)=\frac{\sqrt{2} \int_{0}^{\frac{1}{2}} \frac{\operatorname{Re}[G(\epsilon, \kappa, \xi)] d \kappa}{1-v G(\epsilon, \kappa)}}{\sqrt{-\int_{0}^{\frac{1}{2}} \frac{1}{(1-v G(\epsilon, \kappa))^{2}} \frac{\partial G}{\partial \epsilon}(\epsilon, \kappa) d \kappa}} .
$$

\section{Resultados}

\subsection{Resultados numéricos para os níveis de energia}

Na Figura 1 apresentamos os níveis de energia dos estados localizados para $\alpha=2$ e $\alpha=1.6$. Com exceção do poço na origem, a intensidade dos poços quânticos é dada por $v=-0.2$. Na origem, a intensidade, denotada por $v_{0}$, assumirá outros valores. No painel (a), em que $v_{0} / v>1$, se destaca a existência de apenas um nível de estado localizado. Este se encontra abaixo da banda inferior, $j=0$. No painel (b), em que $v_{0} / v<1$, há um nível de energia abaixo da banda inferior. Entretanto, há um estado localizado em cada um dos gaps (faixas de energia proibida). No painel (c), em que $v_{0} / v>1$, a distância entre o nível mais baixo do estado localizado e o fundo da banda inferior aumenta ao passar da ordem $\alpha=2.0$ para $\alpha=1.6$. No painel (d), a distância entre o nível mais baixo e o topo da banda inferior aumenta ao passar de $\alpha=2.0$ para $\alpha=1.6$. 

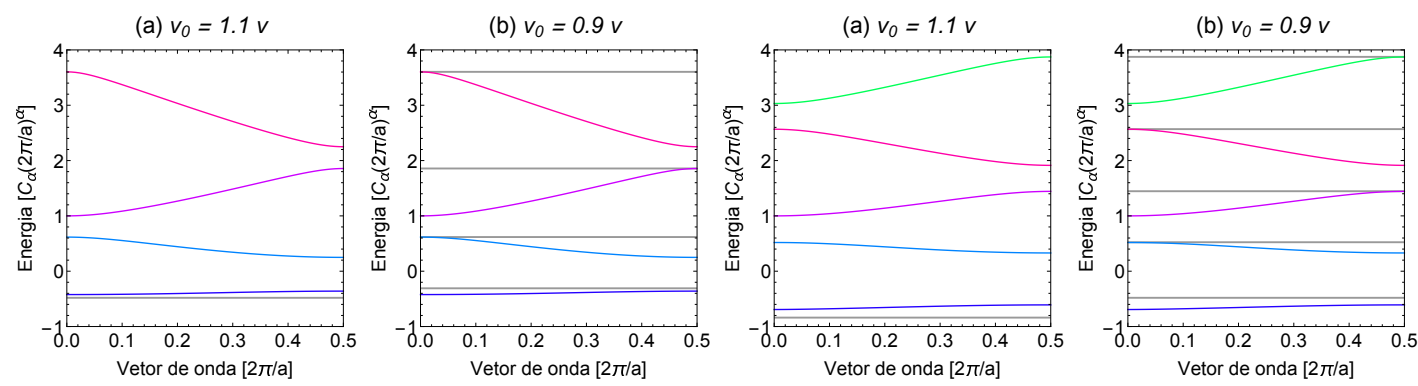

Figura 1: Gráfico dos níveis de energia dos estados localizados com derivada de ordem $\alpha=2.0$ em (a) e (b) e $\alpha=1.6$, em (c) e (d). A intensidade do potencial na origem é $v_{0}=1.1 v$ em (a) e (c) e $v_{0}=0.9 v$, em (b) e (d).

\subsection{Função de onda e seu comportamento assintótico}

Na Figura 2 apresentamos as funções de onda do primeiro nível de energia dos estados localizados calculados para $\alpha=2.0$ e 1.6. As funções de onda são simétricas com relação ao poço quântico da origem e estão bem localizadas dentro da janela selecionada. Além disso, as funções apresentam singularidades nos poços quânticos. No painel (a), a função não se anula e possui o mesmo sinal nos diferentes poços quânticos. No painel (b) a função se anula entre os poços quânticos e apresenta alternância de sinal de poço para poço. Nos painéis (c) e (d), as figuras apresentam as mesmas características do caso inteiro. Entretanto, as singularidades estão mais acentuadas.
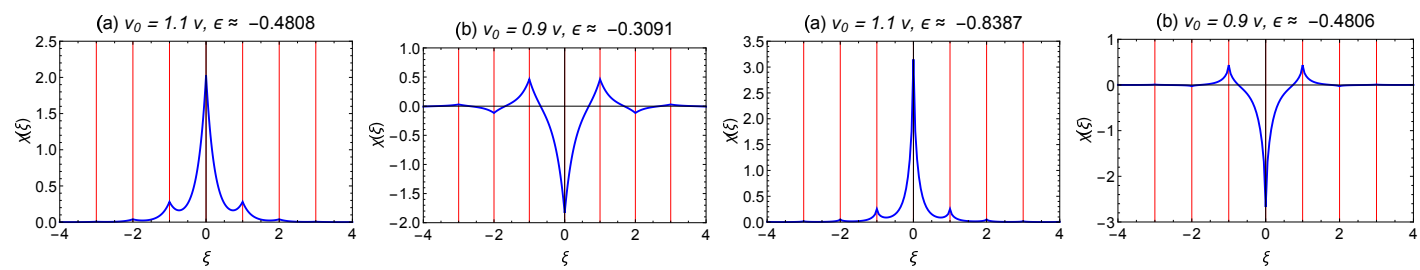

Figura 2: Gráfico da função de onda relativa ao primeiro nível de energia de estado localizado para $\alpha=2.0$, (a) e (b) e para $\alpha=1.6$, (c) e (d). No item (a) e (c) $v_{0}=1.1 v$, no item (b) e (d) $v_{0}=0.9 v$.

As funções de onda, mostradas na Figura 2, apresentam diferentes comportamentos assintóticos. Para $\alpha=2.0$ decaem exponencialmente, enquanto para $\alpha=1.6$ decaem em forma de lei de potência [3].

Analisamos a sequência de valores $\Omega_{n}=|\chi(n)|$, com $n$ inteiro positivo. Se o decaimento de $\Omega_{n}$, quando $n$ tende ao infinito, fosse um produto entre a lei exponencial e a lei de potência, teríamos que

$$
\Omega_{n} \approx \Lambda e^{-h n} n^{-\gamma},
$$

quando $n$ suficientemente grande. Consequentemente,

$$
\ln \left(\Omega_{n} / \Omega_{n+1}\right) \approx h \ln (1+1 / n)+\gamma .
$$


Para estimar os valores de $h$ e $\gamma$, ajustamos uma reta aos pontos de um gráfico $\ln \left(\Omega_{n} / \Omega_{n+1}\right)$ contra $\ln (1+1 / n)$. Os resultados do ajuste para $\alpha=2.0$ e 1.6 são mostrados na Figura 3. No painel (a), para $\alpha=2$, temos $\gamma=0$ e $h \approx 1.97$. Assim, no caso inteiro temos um decaimento puramente exponencial. No painel (b), para $\alpha=1.6$, temos $h=0 \mathrm{e}$ $\gamma \approx 2.614$. Assim, no caso fracionário temos um decaimento em forma de lei de potência e a potência de decaimento é aproximadamente $\gamma=(\alpha+1)$. Este resultado pode ser demonstrado exatamente [3], mediante extensão da função de onda ao plano de valores complexos de $\kappa$.
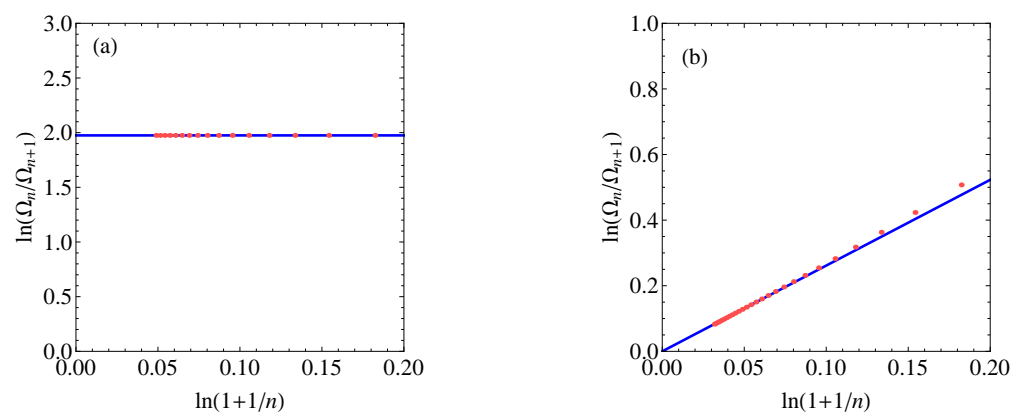

Figura 3: Comportamento assintótico do estado localizado de menor energia no modelo de Kronig-Penney com defeito. Os poços têm intensidade adimensional $v=-0.2 \mathrm{com}$ exceção do poço na origem que tem intensidade $v_{0}=1.1 v$. A ordem da derivada de Riesz é (a) $\alpha=2.0$ e (b) $\alpha=1.6$.

\section{Conclusões}

Pelo método da transformada de Fourier, calculamos os estados localizados do modelo de Kronig-Penney fracionário com defeito, para diferentes valores de intensidades de potencial e para diferentes ordens da derivada fracionária de Riesz: $\alpha=2.0$ e 1.6. Analisamos as funções de onda para o primeiro estado localizado. De um lado, observamos que o decaimento é exponencial para $\alpha=2.0$. Entretanto, para o valor fracionário de $\alpha$, as funções decaem em forma de lei de potência, com expoente $-(\alpha+1)$.

\section{Referências}

[1] S. Jarosz. A Equação de Schrödinger Fracionária com Potenciais Delta, Dissertação de Mestrado, Unicamp, 2016.

[2] E. C. de Oliveira; F. S. Costa and J. Vaz. The fractional Schrödinger equation for delta potentials. Journal of Mathematical Physics, 51:123517, 2010. DOI:10.1063/1.3525976

[3] A. Vellasco-Gomes. Estrutura eletrônica de cristais: Generalização mediante o Cálculo Fracionário, Tese de Doutorado, Unesp, 2018. 